\title{
A decade of sustained geographic spread of HIV infections among women in Durban, South Africa
}

Gita Ramjee ${ }^{1,2,3^{*} \dagger}$, Benn Sartorius ${ }^{4 \dagger}$, Natashia Morris ${ }^{5 \dagger}$, Handan Wand ${ }^{6 \dagger}$, Tarylee Reddy ${ }^{7 \dagger}$, Justin D. Yssel ${ }^{1 \dagger}$ and Frank Tanser ${ }^{4,8,9,10+}$

\begin{abstract}
Background: Fine scale geospatial analysis of HIV infection patterns can be used to facilitate geographically targeted interventions. Our objective was to use the geospatial technology to map age and time standardized HIV incidence rates over a period of 10 years to identify communities at high risk of HIV in the greater Durban area.

Methods: HIV incidence rates from 7557 South African women enrolled in five community-based HIV prevention trials (2002-2012) were mapped using participant household global positioning system (GPS) coordinates. Age and period standardized HIV incidence rates were calculated for 43 recruitment clusters across greater Durban. Bayesian conditional autoregressive areal spatial regression (CAR) was used to identify significant patterns and clustering of new HIV infections in recruitment communities.

Results: The total person-time in the cohort was 9093.93 years and 613 seroconversions were observed. The overall crude HIV incidence rate across all communities was 6.74 per 100PY (95\% Cl: 6.22-7.30). 95\% of the clusters had HIV incidence rates greater than 3 per 100PY. The CAR analysis identified six communities with significantly high HIV incidence. Estimated relative risks for these clusters ranged from 1.34 to 1.70. Consistent with these results, age standardized HIV incidence rates were also highest in these clusters and estimated to be 10 or more per 100 PY. Compared to women $35+$ years old younger women were more likely to reside in the highest incidence areas (aOR: 1.51, 95\% Cl: 1.06-2.15; aOR: 1.59, 95\% Cl: 1.19-2.14 and aOR: 1.62, 95\% Cl: 1.2-2.18 for <20, 20-24, 25-29 years old respectively). Partnership factors ( $2+$ sex partners and being unmarried/not cohabiting) were also more common in the highest incidence clusters (aOR 1.48, 95\% Cl: 1.25-1.75 and aOR 1.54, 95\% Cl: 1.28-1.84 respectively).
\end{abstract}

Conclusion: Fine geospatial analysis showed a continuous, unrelenting, hyper HIV epidemic in most of the greater Durban region with six communities characterised by particularly high levels of HIV incidence. The results motivate for comprehensive community-based HIV prevention approaches including expanded access to PrEP. In addition, a higher concentration of HIV related services is required in the highest risk communities to effectively reach the most vulnerable populations.

Keywords: HIV, Spatial epidemiology, Mapping, Incidence, Risk factors, Heterogeneity

\footnotetext{
* Correspondence: Gita.Ramjee@mrc.ac.za

† Gita Ramjee, Benn Sartorius, Natashia Morris, Handan Wand, Tarylee Reddy, Justin D. Yssel and Frank Tanser contributed equally to this work.

${ }^{1}$ HIV Prevention Research Unit, South African Medical Research Council, 123

Jan Hofmeyr Road, Westville, Durban, KwaZulu-Natal 3630, South Africa

2Department of Epidemiology and Population Health, London School of

Hygiene and Tropical Medicine, London, UK

Full list of author information is available at the end of the article
}

(c) The Author(s). 2019 Open Access This article is distributed under the terms of the Creative Commons Attribution 4.0 International License (http://creativecommons.org/licenses/by/4.0/), which permits unrestricted use, distribution, and reproduction in any medium, provided you give appropriate credit to the original author(s) and the source, provide a link to the Creative Commons license, and indicate if changes were made. The Creative Commons Public Domain Dedication waiver (http://creativecommons.org/publicdomain/zero/1.0/) applies to the data made available in this article, unless otherwise stated. 


\section{Background}

South Africa has over 7 million human immunodeficiency virus (HIV) infected individuals $[1,2]$. HIV prevalence in the country varies greatly across the provinces [3] and is highest in KwaZulu-Natal (KZN). The reasons for the higher burden in KZN (particularly among young women and girls) are multi-faceted and dependent on complex economic and localised social, behavioural and cultural factors [4]. Coupled with high HIV infection rates, is the high prevalence and incidence of other curable sexually transmitted infections (STIs) [5]. Furthermore, factors such as age of sexual debut [6], having a male partner aged 25-34 [7, 8], being unmarried or not living with a partner [9], multiple sexual partners [10, 11], lack of condom use [1], living in a low antiretroviral therapy (ART) coverage area [12] and high mobility [13], have also been reported as contributing factors in a young woman's vulnerability to the risk of HIV acquisition.

Given the persistent high HIV incidence rates, particularly among young women in $\mathrm{KZN}$ [14], it is clear that more effective approaches are required to tackle the epidemic [15-20]. One aspect receiving increasing interest is finer scale geo-spatial analysis of HIV infection patterns to facilitate more appropriate and geographically targeted interventions $[21,22]$. Previous studies have frequently demonstrated the profound impact of HIV clustering on the spread of the disease [23-25]. Furthermore, given the current climate of declining funds and consistent with the global recommendations from the Joint United Nations program on HIV/AIDS (UNAIDS), optimal allocation of the limited resources requires identification of the geographical patterns and clustering of new HIV infections [26]. The HIV Prevention Research Unit (HPRU) of the South African Medical Research Council (SAMRC) had participated in several large-scale HIV prevention trials from 2002 to 2012. The trials have been conducted among several community-based research sites located across the greater Durban region. These trials primarily sought to determine the efficacy of various female initiated HIV prevention options; however, investigational products being tested found no effect on preventing HIV infections [15-19]. The primary objective of this study is to assess the geographical variations and clustering of HIV infections at a localized level in recruitment communities. Results from our study will guide policy makers to develop tailored prevention strategies in order to allocate scarce resources by targeting the most at risk individuals at a sub-geographical level.

\section{Methods}

\section{Study area and population}

From 2002 to 2012, the HPRU of the SAMRC participated in five international multi-centre HIV prevention clinical trials [15-19]. A total of 9145 consenting women were enrolled in this combined cohort over the period of ten years. All consenting women's places of residence (or nearest location point to residence) were geo-coordinated using Geographic Positioning System (GPS) at the time of enrolment and updated during follow-up visits (Additional file 2: Table S1 details the participating communities). We had access to GPS data of 7557 women, which were included in this analysis (Fig. 1).

GPS coordinate data were collected using Garmin ${ }^{\mathrm{Tm}}$ Nüvi (model 2360) handheld devices, downloaded into Microsoft Access and plotted spatially using ArcGIS (version 10.4, CA). A total of 43 community-level recruitment area boundaries were developed based on census delineations [27].

Recruitment of trial participants was from communities in urban (peri-urban) and rural areas. Details of the study population have been described elsewhere [1518]. For the purpose of the present analysis, data for women that were common across the clinical trials was extracted, combined and reported as unidentified and not study or site-specific [15-19, 28].

HIV incidence was the endpoint of all trials included in this analysis. HIV diagnostic testing was conducted using two rapid tests on whole blood sourced from either finger-prick or venepuncture (Determine HIV-1/2, Abbot Laboratories, Tokyo, Japan and Oraquick, Orasure Technologies, Bethlehem, PA, USA). The Abbot IMX Enzyme-linked immunosorbent assay (ELISA) test (Abbot Diagnostics, Africa Division), in combination with the Vironostika HIV1/2 ELISA was used on whole blood sourced from venepuncture for discordant/unequivocal results.

The age eligibility criteria were consistent across all trials ( $>18$ years of age) except for one which enrolled women aged 16 and older. Median age across the trials varied marginally between 24 and 28 years. The average screening to enrolment ratio was $47 \%$ in this combined cohort. Other eligibility criteria were broadly similar for all studies. At each visit, participants received HIV risk reduction counselling, STI testing and treatment and had access to male and/or female condoms. Women who tested HIV positive at screening were referred to local health care facilities for care and support. Women who HIV seroconverted during the trial remained in the study and received ongoing safe sex counselling, STI testing and treatment, and condom provision. All participants provided written informed consent to participate in the studies.

\section{Socio-demographic data collection}

Data pertaining to age, contraceptive use, STI at screening (Chlamydia, Gonorrhoea, Syphilis and Trichomonas) and condom use at last sex act was consistent across all 


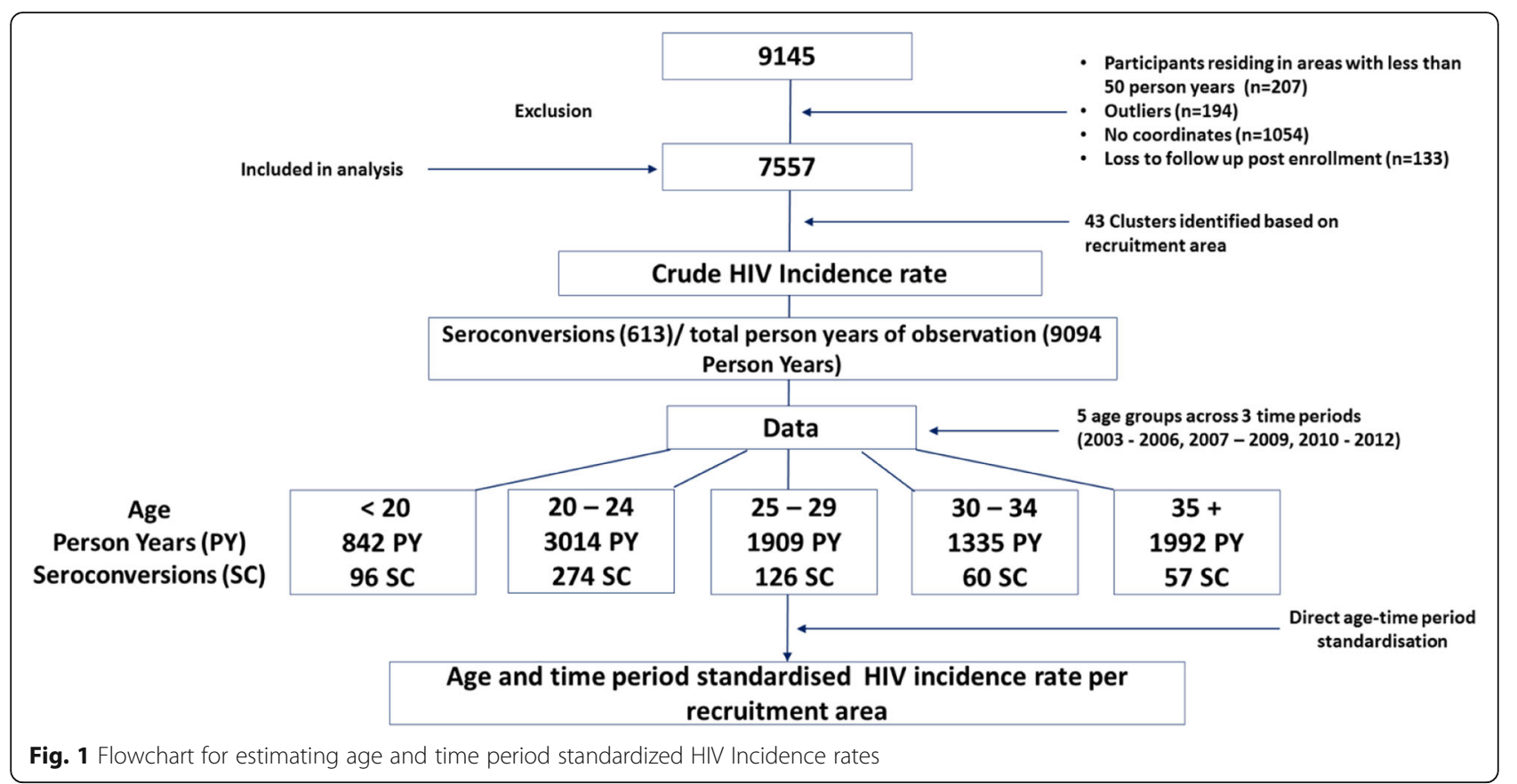

five clinical trials. Four of the clinical trials included marital status/cohabiting and parity data collection. The number of sex partners in the last three months was collected in three of the five clinical trials.

\section{Characterisation of high incidence communities}

Participant data were classified into five age categories $(<20,20-24,25-29,30-34,35+$ years old $)$ across three-time ranges (2003-2006, 2007-2009 and 20102012). HIV incidence rates were calculated in each of fifteen strata ( 5 age groups and 3 time periods) in every recruitment area and a combined weighted estimate for each recruitment area was calculated. We employed direct age-time period standardization to obtain standardized HIV incidence estimates per area to facilitate legitimate geographical comparison (free from the influence of underlying differences in the age-composition of participants in different areas as well as overall incidence changes over time). The reference population was all women enrolled in the HIV prevention trials conducted at HPRU sites between 2002 and 2012.

The characteristics of the study population were compared across pre-defined HIV incidence rate categories $(\leq 5,5-6.9,7-8.9$ and $9+$ per 100 person-year (PY)) as the dependent ordinal variable. This analysis utilised data at the individual women-level within this ordinal variable. Age, marriage/cohabitation, type of contraception used, STI at baseline, number of sexual partners in the last three months and parity data were included as the explanatory variables at the individual level. Univariable and multivariable ordered logistic regression, with recruitment area (community or group of communities) cluster robust standard errors, were used to identify prominent factors associated with higher incidence. Stepwise forward selection regression (inclusion if $p<$ 0.10 ) was used to construct the final multivariable model. Adjusted odds ratios (aOR) and 95\% confidence intervals (CI's) are presented in Table 1 . The proportional odds (or parallel regression) assumption for this modelling approach was checked and upheld (Brant's test) [29].

An aggregate of women's residence per recruitment area representing a minimum of $50 \mathrm{PY}$ were included in HIV incidence rate calculations $(n=7557)$ following exclusion of a) outlying participants not resident within core recruitment areas $(n=194)$, or residing in areas with less than 50 total PY $(n=207)$, (b) participants for whom no GPS data had been collected $(n=1054)$ and (c) participants who did not attend any follow up visits post enrolment $(n=133)$ (Fig. 1). The crude HIV incidence rate across all communities was calculated by dividing the number of seroconversions by total person-years of observation.

\section{Micro-geographical clustering analysis}

We employed the most widely used Bayesian conditional autoregressive (CAR) hierarchical model to assess incidence risk across the 43 areas and mapped the relative risk for each community (Figure 3) [30]. Bayesian hierarchical models are one of the main statistical approaches for making inferences regarding the underlying relative risks of a given disease across often disjointed geographical areas. In addition to the parameters to account for shared boundaries (neighbouring areas) and 
Table 1 Characteristics of the study population by the categories of the HIV incidence rates and multivariable regression analysis

\begin{tabular}{|c|c|c|c|c|c|c|c|}
\hline & Total N (\%) & $\leq 5$ per $100 \mathrm{PY}$ & 5-6.9 per 100PY & 7-8.9 per 100PY & 9+ per 100PY & Adjusted OR & $p$-value \\
\hline \multicolumn{8}{|l|}{ Age group } \\
\hline$<20$ & $768(10 \%)$ & $75(7 \%)$ & $321(11 \%)$ & 199 (10\%) & $173(10 \%)$ & $1.51(1.06-2.15)^{*}$ & 0.023 \\
\hline $20-24$ & $2606(34 \%)$ & 346 (33\%) & 986 (35\%) & 667 (34\%) & 607 (35\%) & $1.59(1.19-2.14)^{*}$ & 0.002 \\
\hline $25-29$ & $1602(21 \%)$ & 239 (23\%) & $576(21 \%)$ & 382 (20\%) & 405 (23\%) & $1.62(1.2-2.18)^{*}$ & 0.001 \\
\hline $30-34$ & $1027(14 \%)$ & $166(16 \%)$ & $386(14 \%)$ & 240 (12\%) & 235 (13\%) & $1.33(0.98-1.82)$ & 0.069 \\
\hline $35+$ & $1554(21 \%)$ & 231 (22\%) & 539 (19\%) & 443 (23\%) & 341 (19\%) & 1 & \\
\hline \multicolumn{8}{|l|}{ Education } \\
\hline None & $3322(55 \%)$ & 577 (56\%) & 1189 (52\%) & $637(50 \%)$ & 919 (60\%) & 1 & \\
\hline Primary & 2485 (41\%) & $436(42 \%)$ & 992 (44\%) & 535 (42\%) & $522(35 \%)$ & $0.8(0.7-0.92)^{*}$ & 0.002 \\
\hline Secondary or higher & $281(5 \%)$ & $19(2 \%)$ & $89(4 \%)$ & $92(7 \%)$ & $81(5 \%)$ & $1.46(1.1-1.95)^{*}$ & 0.009 \\
\hline \multicolumn{8}{|c|}{ Contraceptive use at baseline } \\
\hline None & 1079 (14\%) & $108(10 \%)$ & $416(15 \%)$ & $356(18 \%)$ & 199 (11\%) & 1 & \\
\hline Male/Female condom & $1095(15 \%)$ & $125(12 \%)$ & $386(14 \%)$ & $305(16 \%)$ & $279(16 \%)$ & $1.14(0.65-2.01)$ & 0.639 \\
\hline IUD or Sterile & $619(8 \%)$ & 107 (10\%) & $222(8 \%)$ & $183(10 \%)$ & $107(6 \%)$ & $1.28(0.71-2.29)$ & 0.406 \\
\hline Oral contraceptives & $784(10 \%)$ & $168(16 \%)$ & $250(9 \%)$ & $146(8 \%)$ & $220(13 \%)$ & $1.71(1.02-2.86)^{*}$ & 0.041 \\
\hline Injectables & $3980(53 \%)$ & 549 (52\%) & $1534(55 \%)$ & 941 (49\%) & 956 (54\%) & $1.81(1.1-2.96)^{*}$ & 0.019 \\
\hline \multicolumn{8}{|l|}{ Condom use at last sex } \\
\hline Yes & $4850(66 \%)$ & 704 (70\%) & $1859(68 \%)$ & 1079 (58\%) & $1208(70 \%)$ & & \\
\hline No & $2491(34 \%)$ & $303(30 \%)$ & 877 (32\%) & 792 (42\%) & $519(30 \%)$ & $1.09(0.998-1.19)$ & 0.056 \\
\hline \multicolumn{8}{|l|}{ Any STI } \\
\hline No & $6193(82 \%)$ & $900(85 \%)$ & $2293(82 \%)$ & 1569 (81\%) & 1431 (81\%) & - & \\
\hline Yes & $1356(18 \%)$ & $156(15 \%)$ & $511(18 \%)$ & $361(19 \%)$ & $328(19 \%)$ & $1.04(0.87-1.23)$ & 0.664 \\
\hline \multicolumn{8}{|l|}{ Number of sex partners } \\
\hline 1 & $3929(86 \%)$ & 547 (89\%) & $1491(86 \%)$ & $1108(85 \%)$ & 783 (84\%) & 1 & \\
\hline $2+$ & $661(14 \%)$ & $69(11 \%)$ & $238(14 \%)$ & 199 (15\%) & $155(16 \%)$ & $1.48(1.25-1.75)^{*}$ & $<0.001$ \\
\hline \multicolumn{8}{|l|}{ Parity } \\
\hline 0 & 757 (13\%) & $79(10 \%)$ & $342(13 \%)$ & $170(11 \%)$ & $166(14 \%)$ & $1.23(0.91-1.68)$ & 0.18 \\
\hline 1 & $2610(43 \%)$ & $340(42 \%)$ & $1075(43 \%)$ & $634(43 \%)$ & $561(46 \%)$ & $1.13(0.87-1.46)$ & 0.356 \\
\hline 2 & $1348(22 \%)$ & $212(26 \%)$ & $528(21 \%)$ & $326(22 \%)$ & $282(23 \%)$ & $1.09(0.85-1.39)$ & 0.489 \\
\hline $3+$ & $1317(22 \%)$ & $175(22 \%)$ & $575(23 \%)$ & $358(24 \%)$ & 209 (17\%) & & \\
\hline \multicolumn{8}{|l|}{ Married/Cohabiting } \\
\hline No & 4467 (74\%) & 478 (59\%) & $1890(75 \%)$ & 1145 (77\%) & 954 (78\%) & $1.54(1.28-1.84)^{*}$ & $<0.001$ \\
\hline Yes & $1564(26 \%)$ & 327 (41\%) & $629(25 \%)$ & $344(23 \%)$ & $264(22 \%)$ & 1 & \\
\hline
\end{tabular}

Note:

Age group, education, contraceptive use, number of sex partners and marital status were significant in mulivariable analysis. Adjusted odds ratios for condom use a last sex, STI at screening and parity were computed by adjusting for the aforementioned significant variables

Multivariable ordered logistic regression model based on participants who had complete data for all variables. The likelihood ratio test indicated that there was no violation of the proportional odds assumption in the final ordered logistic regression model that was fitted ( $p$-value 0.100$)$

aOR Adjusted Odds Ratio

IUD Intrauterine device

STI Sexually-transmitted Infection

* Statistically significant ( $p$-value $<0.05$ )

unmeasured heterogeneity within areas, we also included additional covariate terms for age and time period to account for the potential confounding effect of these covariates and how they may vary across the 43 suburbs. We employed a Bayesian conditional autoregressive (CAR) hierarchical model to assess incidence risk across the 43 areas and mapped the relative risk for each community. Bayesian hierarchical models are most commonly used to address the problems posed by small area analysis. We utilised the Besag, York and Molliè (BYM) or convolution CAR model, which is formulated as follows: 


$$
\begin{aligned}
& O_{i \sim \operatorname{Poisson}}\left(E_{i} \gamma_{i}\right) \\
& \log \left(\gamma_{i}\right)=\alpha+\varepsilon_{i}+\phi_{i}
\end{aligned}
$$

where $\gamma_{i}$ is the standardised relative risk in area $\mathrm{i}=1$ to 43, $O_{i}$ is the number of incident HIV events in area i, $E_{i}$ is the expected number of incidence events based on person time contribution, $\varepsilon_{i}$ is the small area unstructured random effect term (to capture unstructured heterogeneity) and $\phi_{i}$ is the CAR spatial term (to capture structured variation). We used an adjacency matrix of common neighbouring areas (shared boundaries) of a given suburb when modelling this parameter whereby $\phi_{\mathrm{i}}$ is the sum of the weighted neighbourhood values; $\varepsilon_{\mathrm{i}}$ was modelled assuming an independent normal distribution $\varepsilon_{i} \sim N\left(0, \sigma_{\varepsilon}^{2}\right)$ with variance $\sigma_{\varepsilon}^{2}$. Non-informative gamma priors were used for variance parameters in both the unstructured and structured random effects.

In addition to the classical parametrization of the model proposed by BYM we also included additional covariate terms for age and period of the individual within the regions 1 to 43 to account for the confounding effect of these covariates and how they may vary across the 43 suburbs. Markov chain Monte Carlo simulation (MCMC) was used to fit this model [30] and implemented in the Bayesian software package WinBUGS [31]. Visual inspection of the parameter series plots was used to assess model convergence as well as by using Gelman-Rubin statistics [32].

\section{Ethics approval and consent to participate}

Ethical approval for the trials were received from the University of KwaZulu-Natal Biomedical Research Ethics Committee and the South African Medical Research Council ethics committee.

\section{Results}

The total person-time in the cohort was 9093.93 years and 613 seroconversions were observed.

\section{Characterisation of the participants}

Demographic characteristics and baseline sexual behaviour of women are presented overall and across the categories of HIV incidence rates (Table 1). The mean and standard deviation of age in the cohort was 27.75 and $7 \cdot 86$, respectively. More than $60 \%$ of the women participants were less than 30 years old. Majority of the women ( $>80 \%$ ) were unmarried and/or not living with their sexual partners and more than half of the women had no education. The most commonly used contraception at baseline were injectables (53\%); while 10 and $15 \%$ of the study population reported using oral contraceptives and male/female condoms respectively. Approximately two thirds of women reported condom-use during their last sex act. At baseline, prevalence of STIs exceeded 18\% in the 5-6.9, 7-8.9 and 9+ incidence categories compared with $15 \%$ in the $\leq 5 \mathrm{HIV}$ incidence category. Most women had already given birth previously; specifically, 43, 22 and $22 \%$ reported having 1, 2 and 3 children respectively.

Compared to the women $35+$ years old, younger women were significantly more likely to be in the highest HIV incidence category (aOR 1.51, 95\% CI: 1.062.15; aOR 1.59, 95\% CI: $1.19-2.14$ and aOR 1.62, 95\% CI: $1.2-2.18$ for $<20,20-24,25-29$ years old respectively). Women who had two or more sex partners were $50 \%$ more likely to be in a higher HIV incidence area compared to women with a single partner (aOR 1.48, 95\% CI:1.25-1.75; $p<0.001)$. A similar finding was observed for women who were unmarried or not cohabiting (aOR 1.54, 95\% CI: 1.28-1.84; $p<0.001$ ).

\section{Micro-geographical clustering analysis}

Estimated relative risks (RRs) from the Bayesian CAR model were estimated across the 43 sub-geographical units and presented in Additional file 1: Figure S1. Our analysis identified six clusters located centrally and in the northern neighbouring areas of Durban (Figure 2). Estimated RRs for these clusters ranged from 1.34 to 1.70. Consistent with these results, age standardized HIV incidence rates were also highest in these clusters and estimated to be as high as 10 to 11 per 100 PY.

\section{Discussion}

Through finer geospatial analysis of our data from over 7000 women participants, we observed a continuous and unrelenting hyper HIV epidemic in the greater Durban area over a ten-year period; with HIV incidence rates ranging from 3 to 12 per 100 woman-year (WY). These high HIV incidence rates were observed among trial participants who received regular HIV pre- and post- test counselling, safe sex counselling, treatment of curable STIs and male and female condom promotion. The high incidence rate observed during this period are similar to that reported from the CAP004 trial [33]; conducted between 2007 and 2009 in Durban. In that trial HIV incidence rates in the placebo group was reported to be $9 /$ 100 PY. Similarly, a cohort study by Nel et al. (20072009) observed an HIV incidence rate of 7.2/100 PY in the greater Durban area [34]. More recent data from the ASPIRE trial [35] showed that HIV incidence rates ranged from 5.4/100py to $8 / 100 \mathrm{PY}$ in the same communities from which this combined data was generated. The ASPIRE trial, suggested a slight decline in HIV incidence in these communities, However, rates as high as $8 / 100 \mathrm{PY}$ are unacceptable given the aggressive roll out 
Clustered study sites (Bayesian CAR model)

Non-clustered study sites: 4.0 to 12.0 per $100 \mathrm{PY}$
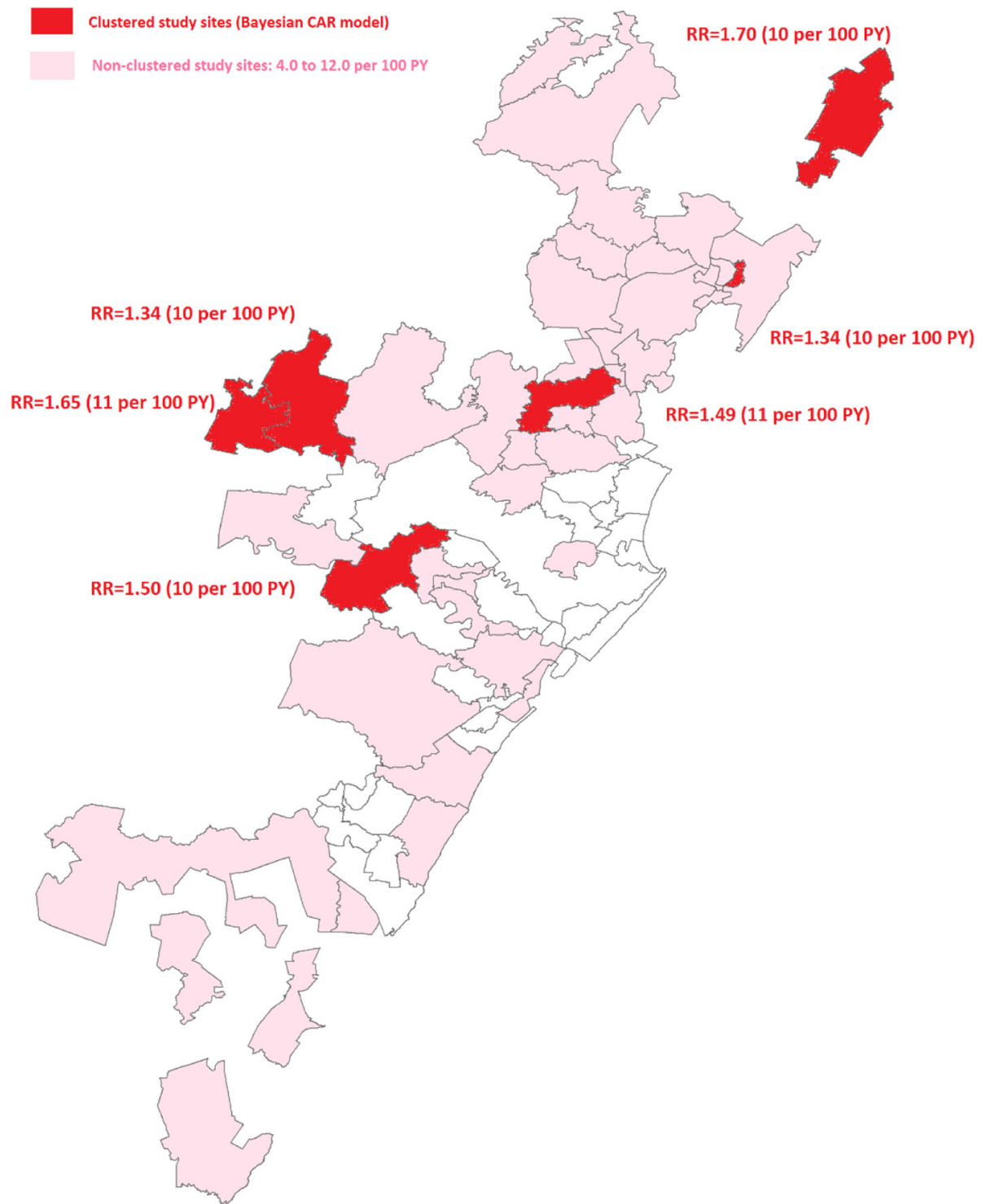

Fig. 2 Relative risks (RRs) from the Bayesian conditional autoregressive (CAR) model and age-period adjusted HIV incidence rates (per 100 PY) for the six clusters with significantly higher incidence rate

of ARV treatment and access to standard prevention options in the country [35].

This is the first comprehensive study to analyse the geographic variation and clustering of HIV incidence rates among women in the greater Durban region. This study has several strengths which overcome many limitations which usually arise with averaged incidence maps (with often low number of events which make robust statistical interference difficult). We employed a Bayesian CAR model, which simultaneously estimates stable spatial and temporal structured patterns and departures from these stable components. In doing so we could capture spatial correlations or unobserved heterogeneity in HIV incidence that cannot be explained by time and other covariates. Results from this analysis underscored several pockets or clusters with high HIV infections in the greater Durban region. These areas were confined to central and northern territories. Previous work conducted in KwaZulu-Natal has examined the micro-geographical patterns of HIV prevalence [36] and HIV incidence in a rural setting over a decade of demographic surveillance [35]. However, no comparative work has been done in an urban setting in Northern KwaZulu-Natal. Similarly, our findings presented prominent heterogeneity in HIV rates in a relatively small geographic extent. In totality, these studies suggest apparent 'corridors' of elevated transmission [37] in the region which, based on the findings herein, may also be 
seen in other urban/peri-urban communities of $\mathrm{KZN}$, as well as other similar hyper-endemic rural populations.

Concurrent with the previous studies, our results also provided additional evidence that these geographically clustered areas could be further differentiated with established risk factors for HIV seroconversion [6-12, 38-40]. As observed in our data, a large number of women, especially younger women, do not seem to be in a stable relationship and are likely to have multiple partners, use barrier methods less frequently thus potentially increasing their risk of HIV acquisition. This was further confirmed by high rates of other curable STIs. These findings are in keeping with those reported previously in KwaZulu-Natal [41]. Research on the role of STIs and inflammation and its impact on the vaginal microbiome and HIV acquisition risk is currently gaining international interest [42-44]. While behavioural and structural risks are important drivers of the HIV acquisition [45], considerable research is being undertaken to understand biological risk of HIV acquisition. It is more likely that in our region, all three risks play a significant role in the risk of HIV acquisition. Consistent with the previous studies, we also showed that women who use injectable and oral contraceptives are at a greater risk of being in a high HIV incidence category. These findings are similar to what we observed in another study [46].

Our results and that of others [41] have consistently shown that younger women are at a greater increased risk of being in the higher HIV incidence categories [47]. More recently, Akullian et al. [8] and de Oliviera et al. [7] showed that when these young women form sexual relationships with men aged 25-34 years of age, they are at particularly high risk for acquiring HIV.

South Africa has the largest ART programme in the world with just over 3.4 million individuals receiving medication [48]. The implementation of universal changes to national policies has been slow with sporadic uptake of ART from HIV positive participants, especially during the earlier period of the trials. This could explain, at least in part, some of the intra-community HIV incidence variability as well as the ubiquitously high rates of infection throughout. In addition, these findings provide further evidence for multiple sub-epidemics within a single region [49] in line with findings from KwaZulu-Natal [37] and other parts of Africa [50-52]. While understanding the spatial structure of HIV burden may allow for an appropriate concentration of services at a micro-geographic level, our data depicts an overall high HIV incidence among women in the entire greater Durban area and calls for a more blanketed and community-wide intervention and prevention efforts to have a major impact in this setting, with a particular focus on addressing the key drivers of the higher incidence identified among this population. In addition to this strategy, using the identified HIV clusters (that may be acting as a node for onward transmission) provides motivation for those communities to receive additional benefit from a more structured and concentrated HIV prevention and intervention approach. Targeting these HIV clusters may have numerous benefits. Firstly, such pockets of HIV are thought to re-seed nearby populations and may concurrently act as a potential "source of attraction" for HIV positive individuals from other communities. Secondly, targeting high risk areas is economically viable especially in resource constrained settings and provides an ideal starting point for ART implementation and scale-up of treatment as prevention (TasP); a strategy that is imperative to break onward HIV transmission. Indeed, without TasP implemented in such HIV concentrated pockets, one may observe a decreased efficacy of existing population-based intervention programmes. Furthermore, it has been previously demonstrated that ART adherence, and indeed uptake, is heavily negatively impacted by geographical barriers. It therefore makes placement of ARV clinics logistical, economical and ethical sense to target vulnerable individuals, aid adherence issues and ultimately achieve maximum penetration of HIV-related services to high-risk areas. This, in addition to a more blanketed intervention and prevention effort, may be the most successful strategy in reducing HIV incidence in a region with a burgeoning HIV epidemic.

The fact that socio-demographic information was not consistently collected across all trials, does pose a limitation and highlights the issue of missing data. A key issue in the extrapolation of data from clinical trials is the generalisability of the incidence data to women in the same geographical area. This may be a limitation of the study where we report on women, mostly of child-bearing age, that participated in a HIV prevention clinical trial. These women may perceive their risk for HIV as higher than the general population, due to individual circumstances and experiences. However, we utilised a consistent set of inclusion and exclusion criteria to enrol women across the decade of clinical trials. This consistency in eligibility (combined with the age and period standardisation approach employed) means that we are comparing a similar sample of women in all areas across time and space. It is thus unlikely that such selection effects could account for the remarkable spatial heterogeneity observed.

\section{Conclusion}

Despite this unfortunate and rather unwelcome result, moving forward there is a clear need to intensify HIV treatment and prevention initiatives in a two-pronged approach. Firstly, due to the unacceptably high HIV incidence in all communities in Durban, an aggressive 
approach to combination prevention strategies including highly effective pre-exposure prophylaxis (PrEP) is needed. Although PrEP is registered for HIV prevention in the country, the roll-out programs are focussed on high risk groups such as men who have sex with men (MSM), sex workers and students. We make a case for universal roll out of PrEP in the greater Durban region with focus on individual risk assessment and provision and monitoring through innovative community-based programs. Secondly, those communities which are clear clustered transmission nodes, need a greater concentration of HIV related services (such as increased availability of HIV/STI clinics, access to treatment), where individuals at higher risk are targeted, and ultimately act as a powerful adjunct to prevention efforts to have a major impact in this setting.

\section{Additional files}

Additional file 1: Figure S1. Community level HIV incidence rates using Bayesian conditional autoregressive (CAR) areal spatial regression analysis. (DOCX $313 \mathrm{~kb}$ )

Additional file 2: Table S1. Description of studies included in cluster analysis detection. (DOCX $14 \mathrm{~kb}$ )

\section{Abbreviations}

aOR: Adjusted odds ratios; ART: Antiretroviral therapy; BYM: Besag, York and Molliè; CAR: Conditional autoregressive; Cl: Confidence interval;

ELISA: Enzyme-linked immunosorbent assay; HIV: Human immunodeficiency virus; HPRU: IV Prevention Research Unit; KZN: KwaZulu-Natal; MCMC: Markov chain Monte Carlo simulation; MSM: Men who have sex with men; PrEP: Preexposure prophylaxis; PY: Person-year; RRs: Relative risks; SAMRC: South African Medical Research Council; STI: Sexually transmitted infections; TasP: Treatment as prevention; UNAIDS: Joint United Nations program on HIV/AIDS; WY: Woman-year

\section{Acknowledgements}

We would like to thank Neetha Morar and Suri Moonsamy for assistance of GPS data management as well as Reshmi Dassaye for review and finalization of this manuscript. Our thanks to study participants and communities who contributed to this study through participation in clinical trials.

\section{Funding}

The trials where these data were generated were supported by various sponsors [Grant Numbers 21082, G0100137, U01Al048008, U01Al069422 and CB04.106G-7]. The funders of the parent studies had no role in the study design, data collection, data analysis, data interpretation or writing of this report. The corresponding author was a principal investigator/investigator of the trials and had access to the site-specific data and had final responsibility for the decision to submit for publication. The analysis for the present study was supported by the South African Medical Research Council.

\section{Availability of data and materials}

The datasets used and/or analysed during the current study are available from the corresponding author on reasonable request.

\section{Authors' contributions}

GR designed the study, aided in manuscript writing and critically reviewed and edited the manuscript. NM trained field teams, managed GPS data and generated the standardised and cluster maps. HW ideated statistical methodology and created the map. TR performed statistical analysis, data interpretation and generation of tables. JDY performed literature searches and drafted the manuscript. BS performed Bayesian CAR analysis and critically reviewed the manuscript. FT oversaw the statistical and spatial analysis and contributed by reviewing the manuscript. All authors have read and approved of this manuscript.

\section{Ethics approval and consent to participate}

All study protocols and informed consent forms were approved by the Biomedical Research Ethics Committee at the University of KwaZulu-Natal as well as the various study-specific Institutional Review Boards. Ethical approval for the trials were received from the University of KwaZulu-Natal Biomedical Research Ethics Committee and the South African Medical Research Council. All participants provided written informed consent to participate in the studies.

\section{Consent for publication}

Not applicable. No individual person's data is presented within this manuscript.

\section{Competing interests}

All authors declare that they have no competing interests.

\section{Publisher's Note}

Springer Nature remains neutral with regard to jurisdictional claims in published maps and institutional affiliations.

\section{Author details}

${ }^{1}$ HIV Prevention Research Unit, South African Medical Research Council, 123 Jan Hofmeyr Road, Westville, Durban, KwaZulu-Natal 3630, South Africa. ${ }^{2}$ Department of Epidemiology and Population Health, London School of Hygiene and Tropical Medicine, London, UK. ${ }^{3}$ School of Medicine, Department of Global Health, University of Washington, Seattle, WA, USA. ${ }^{4}$ School of Nursing and Public Health, University of KwaZulu-Natal, Kwazulu-Natal, Durban, South Africa. ${ }^{5}$ Biostatistics Unit: GIS, South African Medical Research Council, Durban, KwaZulu-Natal, South Africa. ${ }^{6}$ Kirby Institute, University of New South Wales, Kensington, NSW 2052, Australia. ${ }^{7}$ Biostatistics Unit, South African Medical Research Council, Durban, KwaZulu-Natal, South Africa. ${ }^{8}$ Africa Health Research Institute, Durban, Kwazulu-Natal, South Africa. ${ }^{9}$ Research Department of Infection \& Population Health, University College London, London, UK. ${ }^{10}$ Centre for the AIDS Programme of Research in South Africa - CAPRISA, University of KwaZulu-Natal, Durban, Congella, South Africa.

Received: 2 July 2018 Accepted: 13 May 2019

Published online: 07 June 2019

\section{References}

1. Shisana O, Rehle T, Simbayi L, et al. South African national HIV prevalence, incidence and behaviour survey. Cape Town: HSRC Press; 2012. p. 2014

2. HIV/AIDS JUNPO, UNAIDS D. Geneva; 2017. http://www.unaids.org/sites/ default/files/media_asset/UNAIDS_FactSheet_en.pdf. Accessed 30 June 2017

3. Kleinschmidt I, Pettifor A, Morris N, MacPhail C, Rees H. Geographic distribution of human immunodeficiency virus in South Africa. Am J Trop Med Hyg. 2007;77(6):1163-9.

4. Hallman K. Gendered socioeconomic conditions and HIV risk behaviours among young people in South Africa. Afr J AIDS Res. 2005;4(1):37-50.

5. Naidoo S, Wand H, Abbai NS, Ramjee G. High prevalence and incidence of sexually transmitted infections among women living in Kwazulu-Natal, South Africa. AIDS Res Ther. 2014:11(1):31.

6. Wand H, Ramjee G. The relationship between age of coital debut and HIV seroprevalence among women in Durban, South Africa: a cohort study. BMJ Open. 2012;2(1):e000285

7. De Oliveira T, Kharsany AB, Gräf T, Cawood C, Khanyile D, Grobler A, et al. Transmission networks and risk of HIV infection in KwaZulu-Natal, South Africa: a community-wide phylogenetic study. Lancet HIV. 2017;4(1):e41-50.

8. Akullian A, Bershteyn A, Klein D, Vandormael A, Bärnighausen T, Tanser F. Sexual partnership age pairings and risk of HIV acquisition in rural South Africa. AIDS (London, England). 2017;31(12):1755

9. Ramjee G, Moonsamy S, Abbai NS, Wand H. Individual and population level impact of key HIV risk factors on HIV incidence rates in Durban, South Africa. PLoS One. 2016;11(4):e0153969. 
10. Dunkle KL, Jewkes RK, Brown HC, Gray GE, Mclntryre JA, Harlow SD. Genderbased violence, relationship power, and risk of HIV infection in women attending antenatal clinics in South Africa. Lancet. 2004;363(9419):1415-21.

11. Tanser F, Bärnighausen T, Hund L, Garnett GP, McGrath N, Newell M-L. Effect of concurrent sexual partnerships on rate of new HIV infections in a highprevalence, rural south African population: a cohort study. Lancet. 2011; 378(9787):247-55

12. Tanser F, Bärnighausen T, Grapsa E, Zaidi J, Newell M-L. High coverage of ART associated with decline in risk of HIV acquisition in rural KwaZulu-Natal, South Africa. Science. 2013;339(6122):966-71.

13. Dobra A, Bärnighausen T, Vandormael A, Tanser F. Space-time migration patterns and risk of HIV acquisition in rural South Africa. AIDS (London, England). 2017;31(1):137

14. Abaasa A, Crook A, Gafos M, Anywaine Z, Levin J, Wandiembe S, et al. Longterm consistent use of a vaginal microbicide gel among HIV-1 serodiscordant couples in a phase III clinical trial (MDP 301) in rural south-West Uganda. Trials. 2013;14(1):33.

15. Padian NS, van der Straten A, Ramjee G, Chipato T, de Bruyn G, Blanchard K, et al. Diaphragm and lubricant gel for prevention of HIV acquisition in southern African women: a randomised controlled trial. Lancet. 2007; 370(9583):251-61.

16. McCormack S, Ramjee G, Kamali A, Rees H, Crook AM, Gafos M, et al. PRO2000 vaginal gel for prevention of HIV-1 infection (microbicides development Programme 301): a phase 3, randomised, double-blind, parallel-group trial. Lancet. 2010;376(9749):1329-37.

17. Skoler-Karpoff S, Ramjee G, Ahmed K, Altini L, Plagianos MG, Friedland B, et al. Efficacy of Carraguard for prevention of HIV infection in women in South Africa: a randomised, double-blind, placebo-controlled trial. Lancet. 2008, 372(9654):1977-87

18. Marrazzo JM, Ramjee G, Richardson BA, Gomez K, Mgodi N, Nair G, et al. Tenofovir-based preexposure prophylaxis for HIV infection among African women. N Engl J Med. 2015;372(6):509-18.

19. Karim SSA, Richardson BA, Ramjee G, Hoffman IF, Chirenje ZM, Taha T, et al. Safety and effectiveness of BufferGel and 0.5\% PRO2000 gel for the prevention of HIV infection in women. AIDS (London, England). 2011;25(7):957.

20. Gupta GR, Parkhurst JO, Ogden JA, Aggleton P, Mahal A. Structural approaches to HIV prevention. Lancet. 2008;372(9640):764-75.

21. Weir SS, Pailman C, Mahlalela X, Coetzee N, Meidany F, Boerma JT. From people to places: focusing AIDS prevention efforts where it matters most. Aids. 2003;17(6):895-903.

22. Msisha WM, Kapiga SH, Earls FJ, Subramanian S. Place matters: multilevel investigation of HIV distribution in Tanzania. AIDS (London, England). 2008; 22(6):741.

23. Brenner BG, Roger M, Stephens D, Moisi D, Hardy I, Weinberg J, et al. Transmission clustering drives the onward spread of the HIV epidemic among men who have sex with men in Quebec. J Infect Dis. 2011;204(7): 1115-9.

24. Zulu LC, Kalipeni E, Johannes E. Analyzing spatial clustering and the spatiotemporal nature and trends of HIV/AIDS prevalence using GIS: the case of Malawi, 1994-2010. BMC Infect Dis. 2014;14(1):285.

25. Webb D. Mapping the AIDS pandemic: geographical progression of HIV in South Africa 1990-93. NURSING RSA. 1994;9(9):20-1.

26. HIV/AIDS JUNPO. Location, location: connecting people faster to HIV services. Geneva: UNAIDS; 2013. http://www.unaids.org/sites/default/files/ media_asset/LocationLocation_en.pdf. Accessed 1 July 2017.

27. Statistical Release - Statistics South Africa. 2011. https://www.statssa.gov.za/ publications/P03014/P030142011.pdf. Accessed 1 July 2017.

28. Thigpen MC, Kebaabetswe PM, Paxton LA, Smith DK, Rose CE, Segolodi TM, et al. Antiretroviral preexposure prophylaxis for heterosexual HIV transmission in Botswana. N Engl J Med. 2012;2012(367):423-34.

29. Brant R. Assessing proportionality in the proportional odds model for ordinal logistic regression. Biometrics. 1990;46(4):1171-8.

30. Diggle PJ. Statistical analysis of spatial and spatio-temporal point patterns: CRC Press; 2013

31. Lunn DJ, Thomas A, Best N, Spiegelhalter D. WinBUGS-a Bayesian modelling framework: concepts, structure, and extensibility. Stat Comput. 2000;10(4):325-37.

32. Gelman A, Rubin DB. Inference from iterative simulation using multiple sequences. Stat Sci. 1992:457-72.

33. Karim QA, Karim SSA, Frohlich JA, Grobler AC, Baxter C, Mansoor LE, et al. Effectiveness and safety of tenofovir gel, an antiretroviral microbicide, for the prevention of HIV infection in women. Science. 2010;329(5996):1168-74.
34. Nel A, Mabude Z, Smit J, Kotze P, Arbuckle D, Wu J, et al. HIV incidence remains high in KwaZulu-Natal, South Africa: evidence from three districts. PLoS One. 2012;7(4):e35278.

35. Baeten JM, Palanee-Phillips T, Brown ER, Schwartz K, Soto-Torres LE, Govender $\mathrm{V}$, et al. Use of a vaginal ring containing dapivirine for HIV-1 prevention in women. N Engl J Med. 2016;375(22):2121-32.

36. Tanser F, Hosegood V, Bärnighausen T, Herbst K, Nyirenda M, Muhwava W, et al. Cohort profile: Africa Centre demographic information system (ACDIS) and population-based HIV survey. Int J Epidemiol. 2007;37(5):956-62.

37. Tanser F, Bärnighausen T, Dobra A, Sartorius B. Identifying 'corridors of HIV transmission' in a severely affected rural south African population: a case for a shift toward targeted prevention strategies. Int J Epidemiol. 2017;1: 47(2)537-49.

38. Ramjee G, Williams B, Gouws E, Van Dyck E, De Deken B, Karim SA. The impact of incident and prevalent herpes simplex virus-2 infection on the incidence of HIV-1 infection among commercial sex workers in South Africa. JAIDS J Acquir Immune Defic Syndr. 2005:39(3):333-9.

39. Frohlich J, Abdool Karim Q, Mashego M, Sturm A, Abdool Karim S. Opportunities for treating sexually transmitted infections and reducing HIV risk in rural South Africa. J Adv Nurs. 2007:60(4):377-83.

40. Wilkinson D, Connolly A-M, Harrison A, Lurie M, Karim SA. Sexually transmitted disease syndromes in rural South Africa: results from health facility surveillance. Sex Transm Dis. 1998;25(1):20-3.

41. Mlisana K, Naicker N, Werner L, Roberts L, Van Loggerenberg F, Baxter C, et al. Symptomatic vaginal discharge is a poor predictor of sexually transmitted infections and genital tract inflammation in high-risk women in South Africa. J Infect Dis. 2012;206(1):6-14.

42. Klatt NR, Cheu R, Birse K, Zevin AS, Perner M, Noël-Romas L, et al. Vaginal bacteria modify HIV tenofovir microbicide efficacy in African women. Science. 2017;356(6341):938-45.

43. Liebenberg LJ, Masson L, Arnold KB, Mckinnon LR, Werner L, Proctor $E$, et al. Genital — systemic chemokine gradients and the risk of HIV Acquisition in Women. J Acquir Immune Defic Syndr (1999). 2017:74(3):318.

44. Jaumdally SZ, Jones HE, Hoover DR, Gamieldien H, Kriek JM, Langwenya N, et al. Comparison of sampling methods to measure HIV RNA viral load in female genital tract secretions. Am J Reprod Immunol. 2017;77(3).

45. Jewkes RK, Dunkle K, Nduna M, Shai N. Intimate partner violence, relationship power inequity, and incidence of HIV infection in young women in South Africa: a cohort study. Lancet. 2010;376(9734):41-8.

46. Mugo NR, Heffron R, Donnell D, Wald A, Were EO, Rees H, et al. Increased risk of HIV-1 transmission in pregnancy: a prospective study among African HIV-1 serodiscordant couples. AIDS (London, England). 2011;25(15):1887.

47. Karim QA, Kharsany AB, Frohlich JA, Werner L, Mashego M, Mlotshwa M, et al. Stabilizing HIV prevalence masks high HIV incidence rates amongst rural and urban women in KwaZulu-Natal, South Africa. Int J Epidemiol. 2010; 40(4):922-30.

48. South African National Department of Health. Annual report. 2016. www. health.gov.za/index.php/2014-08-15-12-56-31?download...annual-report2016. Accessed 2 July 2017.

49. Tanser F, de Oliveira T, Maheu-Giroux M, Bärnighausen T. Concentrated HIV sub-epidemics in generalized epidemic settings. Curr Opin HIV AIDS. 2014; $9(2): 115$.

50. Wand H, Whitaker C, Ramjee G. Geoadditive models to assess spatial variation of HIV infections among women in local communities of Durban, South Africa. Int J Health Geogr. 2011:10(1):28.

51. Wand H, Ramjee G. Targeting the hotspots: investigating spatial and demographic variations in HIV infection in small communities in South Africa. J Int AIDS Soc. 2010;13(1):41.

52. Cuadros DF, Awad SF, Abu-Raddad LJ. Mapping HIV clustering: a strategy for identifying populations at high risk ofHIV infection in sub-Saharan Africa. Int J Health Geogr. 2013:12(1):28. 